\title{
Can we improve the accuracy of electrocardiographic algorithms for accessory pathway location in children?
}

\author{
Paola Ferrari', Giovanni Malanchini ${ }^{1}$, Marco Racheli ${ }^{1}$, Gabriele Ferrari' , Cristina Leidi ${ }^{1}$, Michele Senni², \\ Paolo Della Bella ${ }^{3}$, Maurizio Malacrida ${ }^{4}$, Simone Gulletta ${ }^{3 *}$, Paolo De Filippo ${ }^{1 *}$ \\ 'Cardiac Electrophysiology and Pacing Unit, Papa Giovanni XXIII Hospital, Bergamo, Italy \\ ${ }^{2}$ Cardiology Division, Cardiovascular Department, Papa Giovanni XXIII Hospital, Bergamo, Italy \\ ${ }^{3}$ Arrhythmia Unit and Electrophysiology Laboratories, San Raffaele Scientific Institute, Milan, Italy \\ ${ }^{4}$ Boston Scientific, Milan, Italy \\ *Both authors equally contributed to the study
}

\section{Editorial}

by Baranchuk et al.

Correspondence to:

Giovanni Malanchini, MD Cardiac Electrophysiology and Pacing Unit, Papa Giovanni XXIII Hospital,

Piazza OMS 1, 24127 Bergamo,

Italy,

phone: +39035 2674674 ,

e-mail: gmalanchini@asst-pg23.it

Copyright by the Author(s), 2022

DOI: 10.33963/KP.a2021.0167

Received:

July 28, 2021

Accepted:

November 28, 2021

Early publication date:

December 1, 2021

\section{A B S T R A C T}

Background: Predicting an accessory pathway location is extremely important in pediatric patients. Aims: We designed a study to compare previously published algorithms by Arruda, Boersma, and Chiang.

Methods: This multicenter study included patients who had undergone successful ablation of one accessory pathway. Analysis of resting 12-lead electrocardiograms was carried out. An aggregated prediction score was constructed on the basis of algorithm agreement, and a structured workflow approach was proposed.

Results: The total population was 120 patients (mean age, $12.7[ \pm 3.6]$ years). The algorithm by Boersma had the highest accuracy (71.7\%). The inter-rater agreement among the 3 reference algorithms, according to left-sided accessory pathway (AP) identification, was good between Boersma and Chiang ( $\mathrm{K}=0.611 ; 95 \%$ confidence interval $[\mathrm{Cl}], 0.468-0.753$ ) but moderate between Arruda and Chiang and between Arruda and Boersma $(\mathrm{K}=0.566 ; 95 \% \mathrm{Cl}, 0.419-0.713$ and $\mathrm{K}=0.582 ; 95 \%$ $\mathrm{Cl}, 0.438-0.727$, respectively). Regarding locations at risk of atrioventricular (AV) block, agreement was fair between Arruda and Chiang and between Boersma and Chiang $(\mathrm{K}=0.358 ; 95 \% \mathrm{Cl}$, $0.195-0.520$ and $\mathrm{K}=0.307 ; 95 \% \mathrm{Cl}, 0.192-0.422$, respectively) but moderate between Arruda and Boersma ( $\mathrm{K}=0.45 ; 95 \% \mathrm{Cl}, 0.304-0.597$ ). On applying a first-step diagnostic evaluation, when concordance was achieved, we were able to correctly identify left-sided or non-left-sided ablation sites in $96.4 \%(n=80)$ of cases. When concordance was achieved, correct prediction of risk/no risk of AV block was achieved in $92.2 \%(n=59)$ of cases.

Conclusions: An aggregated prediction score based on 3 reference algorithms proved able to predict an accessory pathway location very precisely and could be used to plan safely invasive procedures.

Key words: pre-excitation, WPW, algorithm, pediatric, children

\section{INTRODUCTION}

Catheter ablation is now considered the treatment of choice for patients with Wolff-Parkinson-White syndrome. It is successful in more than $95 \%$ of patients, has a relatively low complication rate, and obviates pharmacological side effects [1]. Ablative procedures can be distinguished in terms of their technical peculiarity according to the anatomical location of accessory pathways (AP): the left free wall, the right free wall, anteroseptal, mid-septal, and postero-septal. European guidelines rec- 
WHAT'S NEW?

An aggregated prediction score based on three reference algorithms can predict an accessory pathway location at risk of heart block or the need for left heart catheterization very precisely and could be used to safely plan invasive procedures in pediatric patients.

ommend discussing the potential risks and benefits before performing ablation of an anteroseptal or mid-septal AP, especially the risk of complete heart block [1]. In addition, left-sided AP ablation carries the inherent risk of left heart catheterization, which is especially relevant in pediatric patients [2,3]. To achieve a good prediction of procedural risks connected to the site of AP ablation, various methods have been developed and are of seminal importance in daily practice [4-11]. The algorithms developed are inherently limited by anatomical variability, intrinsic electrocardiogram (ECG) abnormalities, a degree of preexcitation [12], and technical variability in ECG acquisition, especially with regard to precordial leads, and no algorithm seems to have high sensitivity and specificity for all AP locations [13]. While some "reference" algorithms (e.g. those proposed by Arruda et al. [4], Chiang et al. [6] and d'Avila et al. [7]) have displayed similar accuracy in predicting an AP location in the pediatric population [13], the accuracy of seven published algorithms has proved lower than that reported in predicting AP location in adults, especially in identifying right mid-septal or right anteroseptal APs. Overall, the most accurate algorithm reported is that by Boersma et al. $[14,15]$ Therefore, in the absence of conclusive studies $[16,17]$, we designed a study to compare 3 different algorithms (those by Arruda, Boersma, and Chiang - see Supplementary material, Figures S1-3) and to evaluate how an aggregated prediction score based on these algorithms could be useful. This study aimed to assess and compare their accuracy in predicting APs in a large, consecutive multicenter pediatric population, and establish how they could help to safely plan invasive procedures.

\section{METHODS}

\section{Study design}

This multicenter, observational, retrospective study was based on the analysis of resting 12-lead ECGs in children with a ventricular pre-excitation pattern. We screened every consecutive patient referred for electrophysiological study (EPS) who showed a pre-excitation pattern on baseline ECG from January 2013 to June 2019. Patients were enrolled at the ASST Papa Giovanni XXIII Hospital, Bergamo, Italy, and the IRCCS San Raffaele Hospital, Milan, Italy. The study was approved by the Institutional Committee for research of both enrolling institutions. Patients who had undergone successful ablation of one accessory pathway were included in the study. According to a single protocol implemented in both centers, 3 cardiologists experienced in the use of electrocardiographic algorithms for the AP location in children and blinded to the AP location analyzed all resting 12-lead ECGs by using each of the 3 previously published algorithms selected for analysis. In case of discordance, the decision was adjudicated by the majority. The accessory pathway location was adjudicated by the same method reviewing fluoroscopy and electro-anatomical maps. Inclusion criteria were age $<18$ years; structurally normal heart as assessed by prior echocardiogram; at least one AP documentation on invasive EPS; successful catheter ablation at one exact location. Available data were age, weight, height, ECG-predicted location, and exact invasive location of the pathway. As the algorithms by Boersma and Chiang do not provide a specific prediction for pathways located within the coronary sinus $[5,6]$, we excluded patients who had undergone ablation at this site.

\section{Comparison of the three algorithms and the groups of locations}

To show differences between predicted and actual locations of accessory pathways, we considered 13 anatomical regions around the mitral and tricuspid annuli as the possible exact locations, as identified by EPS (Figure 1). We then analyzed the possible locations predicted by each algorithm, which could include one or more of the 13 regions identified. To highlight these differences, we constructed a table (Table 1) indicating the relationship between the predicted locations and the actual ablation sites; the prediction was deemed correct if the pathway was ablated at one site in the same row of Table 1. We also assessed predictions in 3 different regions around the valvular annuli, given the different impact of APs in these regions on the procedure and on risk: we grouped right anterior (RA), right anteroseptal (RAS), and right mid-septal (RMS) pathways, owing to the risk of damage to the His-Purkinje system (at risk of atrioventricular [AV] block), left-sided pathways, due to the need for a trans-septal/trans-aortic approach, and right-sided APs.

\section{Statistical analysis}

Descriptive statistics are reported as means (SD) for normally distributed continuous variables, or medians with $25^{\text {th }}$ to $75^{\text {th }}$ percentiles in the case of skewed distribution. The normality of distribution was tested through the non-parametric Kolmogorov-Smirnov test. Raw exact accuracy of correct prediction of each algorithm was assessed as 


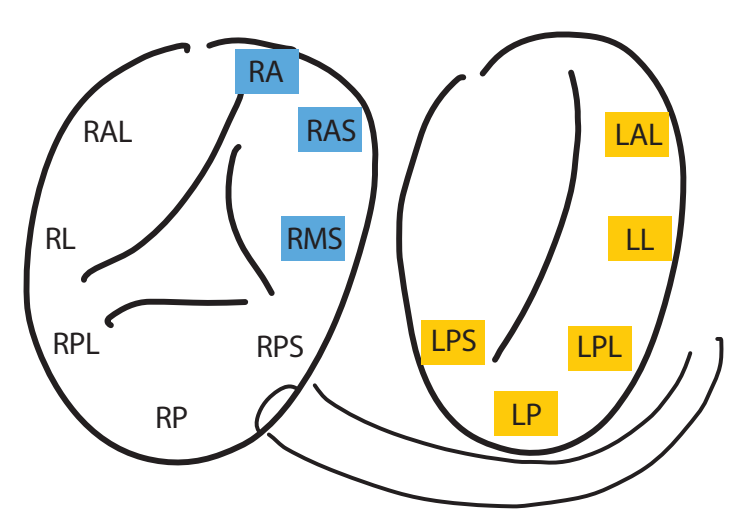

\begin{tabular}{l|c}
\multicolumn{1}{c|}{ AP location } & N (\%) \\
\hline Left lateral (LL) & $29(24)$ \\
Right mid-septal (RMS) & $18(15)$ \\
Right postero-septal (RPS) & $16(13)$ \\
Right antero-septal (RAS) & $15(12)$ \\
Left postero-septal (LPS) & $13(11)$ \\
Left posterolateral (LPL) & $7(6)$ \\
Right lateral (RL) & $6(5)$ \\
Right anterolateral (RAL) & $5(4)$ \\
Right anterior (RA) & $4(3)$ \\
Left posterior (LP) & $3(3)$ \\
Left anterolateral (LAL) & $2(2)$ \\
Right posterior (RP) & $2(2)$ \\
\hline
\end{tabular}

Figure 1. Different sites of accessory pathways grouped according to the risk of atrioventricular block and left-side location. Frequency of different accessory pathway locations according to the site of successful ablation

Abbreviations: AP, accessory pathway

Table 1. Table of matches

\begin{tabular}{|c|c|c|c|c|c|c|}
\hline \multirow{2}{*}{$\begin{array}{l}\text { Successfull } \\
\text { ablation } \\
\text { site }\end{array}$} & \multicolumn{2}{|c|}{ Arruda [4] } & \multicolumn{2}{|c|}{ Boersma [5] } & \multicolumn{2}{|c|}{ Chiang [6] } \\
\hline & $\begin{array}{l}\text { Concordant } \\
\text { algorithm } \\
\text { site }\end{array}$ & $\begin{array}{l}\text { Possible } \\
\text { algorithm } \\
\text { predictions }\end{array}$ & $\begin{array}{l}\text { Concordant } \\
\text { algorithm } \\
\text { site }\end{array}$ & $\begin{array}{l}\text { Possible } \\
\text { algorithm } \\
\text { predictions }\end{array}$ & $\begin{array}{l}\text { Concordant } \\
\text { algorithm } \\
\text { site }\end{array}$ & $\begin{array}{c}\text { Possible } \\
\text { algorithm } \\
\text { predictions }\end{array}$ \\
\hline RA & RA & RA-RAL & AS & RL-AS or AS-PH-RL or RL-PH & RA & RAS-RA \\
\hline RAS & AS & AS & AS & RL-AS or AS-PH-RL or RL-PH & RAS & RAS-RA \\
\hline RMS & MS & MS & MS & RPS-MS & MS & MS \\
\hline RPS & PSTA & PSTA or PSTA-PSMA & RPS/PS & PS or RPS-MS & RPS & RPS \\
\hline RAL & RAL & RA-RAL & $\mathrm{RL}$ & RL-AS or RL-PH or AS-PH-RL & RAL & RAL \\
\hline $\mathrm{RL}$ & $\mathrm{RL}$ & $\mathrm{RL}$ & $\mathrm{RL}$ & RL-AS or RL-PH or AS-PH-RL & $\mathrm{RL}$ & $\mathrm{RL}$ \\
\hline $\mathrm{RP}$ & $\mathrm{RP}$ & RP-RPL & RPS & RPS-MS or PS & $\mathrm{RP}$ & RP-RPL \\
\hline RPL & $\mathrm{RPL}$ & RP-RPL & $\mathrm{RL}$ & RL-AS or RL-PH or AS-PH-RL & RPL & RP-RPL \\
\hline LPS & PSMA & PSTA-PSMA & LPS/PS & LPS & LPS & LPS \\
\hline LAL & LAL & LL-LAL & LL & $\mathrm{LL}$ & LAL & LL-LAL \\
\hline LL & $\mathrm{LL}$ & LL-LAL & LL & LL & $\mathrm{LL}$ & LL-LAL \\
\hline LPL & LPL & LP-LPL & $\mathrm{LL}$ & $\mathrm{LL}$ & LPL & LP-LPL \\
\hline LP & LP & LP-LPL & LPS/LL & LL or LPS & LP & LP-LPL \\
\hline
\end{tabular}

Abbreviations: AS, antero-septal; LAL, left antero-lateral; LL, left lateral; LP, left posterior; LPL, left postero-lateral; LPS, left postero-septal; MS, mid-septal; PH, para-hisian; PSMA, postero-septal mitral annulus; PSTA, postero-septal tricuspid anulus; RA, right anterior; RAL, right antero-lateral; RAS, right antero-septal; RL, right lateral; RMS, right mid-septal; RP, right posterior; RPL, right postero-lateral; RPS, right postero-septal

the percentage of cases correctly assigned to one of the possible predicted locations by each algorithm. Corrected accuracy was calculated by division of raw accuracy the number of possible locations (13.8 and 13 respectively in the Arruda, Boersma, and Chiang algorithms). Bayesian probability $(P[A \mid B])$ considering the prediction of the algorithm attenuated by the "by chance" probability of the AP to be in a locations group was also calculated (Table 3A, B). To evaluate agreement among criteria, an inter-rater agreement statistic $(\mathrm{K})$ was used $[18,19] . \mathrm{K}$ is 1 when there is perfect agreement between the classification algorithms; $\mathrm{K}$ is 0 when the agreement is no better than chance; when grading concordance if $0.21<\mathrm{K}<0.4$, it is considered fair, when $0.41<\mathrm{K}<0.6$, it is considered moderate; when $0.61<\mathrm{K}$ $<0.8$, it is considered good; when $\mathrm{k}>0.81$, it is considered very good; $\mathrm{k}$ is negative when the agreement is worse than chance. An aggregated prediction score was constructed on the basis of criteria agreement, and a structured work- flow approach was proposed. A $P$-value $<0.05$ was considered significant for all tests. All statistical analyses were performed using STATISTICA software, version 7.1 (StatSoft Inc., Tulsa, OK, USA).

\section{RESULTS}

\section{Study population}

From the screened population of 165 patients, 37 were excluded because they did not undergo ablation, and 8 because the accessory pathway was ablated within the cardiac venous system. The final population, therefore, comprised 120 patients. Their mean age was 12.7 (3.6) years, and their mean weight and height were 48.3 (17.1) $\mathrm{kg}$ and $155.6(19.3) \mathrm{cm}$, respectively. The distribution of AP locations is shown in Figure 1. The most frequent location of AP was left lateral (LL), followed by RMS; these two regions, together with the right postero-septal (RPS) location, ac- 
Table 2. Overall raw accuracy and pre-test probability-corrected diagnostic accuracy of the three algorithms in predicting the exact location of accessory pathways

\begin{tabular}{l|c|cc} 
& Arruda [4] & Boersma [5] & Chiang [6] \\
Number of locations & 13 & 8 & 13 \\
Total accuracy & 0.617 & 0.717 & 0.533 \\
Accuracy per group & 0.833 & 0.841 & 0.675 \\
Accuracy left vs. right & 0.933 & 0.833 & 0.85 \\
Corrected accuracy per locations & 8.0158 & 5.736 & 6.929 \\
\hline
\end{tabular}

Table 3. A. Accuracy in locating high-septal, right- and left-sided accessory pathways. Since $31.03 \%$ of APs are located in the anterior, anteroseptal, or mid-septal part of the tricuspid annulus, the probability of a prediction by the algorithm of Arruda being in one of these regions is $64.31 \%$. The same applies to the Boersma algorithm; when it locates the pathway in the high-septal region, the probability that the pathway is located there is $49.31 \%$. B. Accuracy in locating right- vs. left-sided accessory pathways

\begin{tabular}{|c|c|c|c|c|c|c|c|}
\hline $\mathbf{A}$ & & \multicolumn{2}{|c|}{ Arruda [4] } & \multicolumn{2}{|c|}{ Boersma [5] } & \multicolumn{2}{|c|}{ Chiang [6] } \\
\hline Group & $\mathbf{P}$ & TP & $P(A \mid B)$ & TP & $P(A \mid B)$ & TP & $P(A \mid B)$ \\
\hline High sept & $31.3 \%$ & 0.7297 & $64.31 \%$ & 0.8918 & $49.31 \%$ & 0.4324 & $72.17 \%$ \\
\hline Right & $22.4 \%$ & 0.862 & $53.11 \%$ & 0.862 & $33.98 \%$ & 0.7241 & $45.27 \%$ \\
\hline Left & $46.3 \%$ & 0.888 & $82.34 \%$ & 0.7962 & $83.61 \%$ & 0.8148 & $83.93 \%$ \\
\hline B & & \multicolumn{2}{|c|}{ Arruda [4] } & \multicolumn{2}{|c|}{ Boersma [5] } & \multicolumn{2}{|c|}{ Chiang [6] } \\
\hline Group & $\mathbf{P}$ & TP & $P(A \mid B)$ & TP & $P(A \mid B)$ & TP & $P(A \mid B)$ \\
\hline Right & $53.7 \%$ & 0.9696 & $91.4 \%$ & 0.8636 & $83.8 \%$ & 0.8787 & $85.3 \%$ \\
\hline Left & $46.3 \%$ & 0.8888 & $95.9 \%$ & 0.7962 & $82.5 \%$ & 0.8148 & $84.5 \%$ \\
\hline
\end{tabular}

Abbreviations: $\mathrm{P}$, pre-test probability; TP, true positives; $\mathrm{P}(\mathrm{A} \mid \mathrm{B})$, Bayesian probability of ablation site of the pathway where it was predicted by the algorithm considering the pre-test probability

counted for more than $50 \%$ of cases. Patients were treated with radiofrequency or cryo-ablation according to operator and center preference. No patient experienced a major complication during or after the procedure.

\section{Diagnostic accuracy according to reference algorithms}

Without any correction, the algorithm by Boersma et al. proved the ablest to predict pathway locations that were found to be concordant with the site of successful AP ablation (Table 2). The location of a pathway in each group was correctly predicted in $83 \%$ of cases by the Arruda algorithm, in $84 \%$ by the Boersma algorithm, and in $67 \%$ by the Chiang algorithm. All 3 algorithms performed very well in discriminating between left-sided and right-sided AP locations. The highest accuracy was achieved by the Arruda algorithm, which displayed 93\% concordance between the predicted side and the actual side of ablation.

Table 3A reports the accuracy of the 3 algorithms in predicting the location of left-sided APs vs. sites at risk for AV block. Pre-test probability (P) was calculated on the basis of the prevalence of accessory pathways in each of 3 groups of locations in our population. The algorithm by Chiang et al. displayed the greatest predictive power: when this algorithm predicted an AP location in one of these regions, there was a $72.17 \%$ chance that the pathway was located in that region. All 3 algorithms displayed a very high predictive power in locating the AP on the left side: $82.35 \%$, $83.61 \%$, and $83.93 \%$ probability for Arruda, Boersma, and Chiang, respectively.
We also calculated the probability of an AP being on the right or the left side (Table 3B), as predicted by each algorithm. The highest probability was achieved by the Arruda algorithm (91.4\% when predicted on the right and 95.9\% when predicted on the left). Similar values were obtained by applying the Boersma and Chiang algorithms.

\section{Agreement among the algorithms}

Figure 2 reports the inter-rater agreement among the 3 reference algorithms according to the identification of left-sided AP (Figure 2A) and the risk of AV block (Figure 2B). With regard to left-sided AP identification, the strength of agreement was good between the Boersma and Chiang algorithms ( $\mathrm{K}=0.611 ; 95 \%$ confidence interval $[\mathrm{Cl}], 0.468-$ 0.753), whereas it was moderate between Arruda and Chiang algorithms as well as between Arruda and Boersma algorithms $(\mathrm{K}=0.566,95 \% \mathrm{Cl}, 0.419-0.713$, and $\mathrm{K}=0.582$; $95 \% \mathrm{Cl}, 0.438-0.727$, respectively). Regarding sites at risk of AV block, the agreement was fairly good between Arruda and Chiang algorithms as well as between Boersma and Chiang algorithms $(\mathrm{K}=0.358 ; 95 \% \mathrm{Cl}, 0.195-0.520$ and $\mathrm{K}=0.307 ; 95 \% \mathrm{Cl}, 0.192-0.422$, respectively), whereas it was moderate between Arruda and Boersma algorithms ( $\mathrm{K}=0.45 ; 95 \% \mathrm{Cl}, 0.304-0.597)$.

\section{Diagnostic accuracy according to aggregated prediction score}

We applied a step-down approach based on the concordance of the 3 algorithms in identifying both left-sided sites and sites at risk of AV block (Figure 3A, B). All 3 algo- 


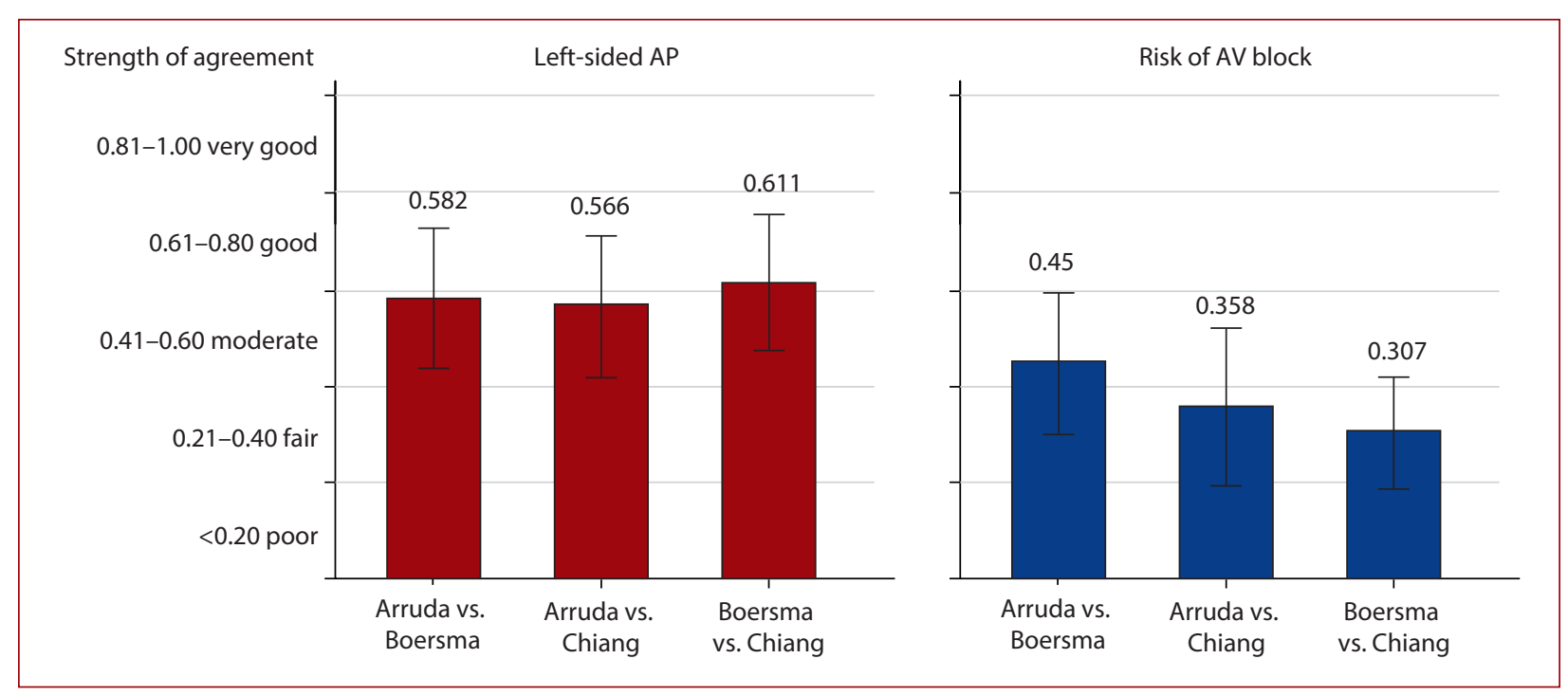

Figure 2. Inter-rater agreement among the three reference algorithms according to the identification of left-sided accessory pathway and the risk of atrioventricular block

Abbreviations: AV block, atrioventricular block; other — see Figure 1

rithms identified the site as left-sided in 38 (31.7\%) cases; 2 algorithms identified it as left-sided in 13 (10.8\%) cases; 1 algorithm identified it as left-sided in 24 (20.0\%) cases, and all algorithms identified the site as non-left-sided in $45(37.5 \%)$ cases. Concordance (all algorithms reported the same result) was found in $69.1 \%(n=83)$ of cases. On applying a first-step diagnostic evaluation when concordance was achieved, we were able to correctly identify left-sided or non-left-sided sites in $96.4 \%(n=80)$ of cases (correctly identified as left-sided: $37 / 38-97.4 \%$; correctly identified as non-left-sided: 43/45 - 95.6\%). Regarding the evaluation of sites at risk of AV block, in 15\% of cases $(n=18)$, all the 3 algorithms identified the predicted site as being at risk of AV block; in $22.5 \%$ of cases ( $n=27), 2$ algorithms identified it as being at risk of AV block; in $24.2 \%$ $(n=29), 1$ algorithm identified it as at risk of AV block, and in 46 (38.3\%) all algorithms identified the site as not being at risk of AV block. Concordance was observed in 53.3\% ( $n=64$ ) of cases. On applying a first-step diagnostic evaluation when concordance was achieved, in 92.2\% ( $n=59)$ of cases we were able to correctly identify sites at risk or not at risk of AV block (correctly identified as being at risk of AV block: 15/18 - 93.3\%; correctly identified as not at risk of AV block: 44/46 — 95.7\%).

\section{DISCUSSION}

The 3 algorithms tested (those introduced by Arruda et al. [4], Boersma et al. [5], and Chiang et al. [6]) showed several differences and were constructed on relatively large populations of patients with ventricular pre-excitation. The sensitivity, specificity, and accuracy reported in the original studies ranged from $90 \%$ to $99 \%$. However, the performance of these algorithms is reported to be reduced in children $[14,15]$. In the present study, the overall accuracy of each of the selected algorithms was found to be higher than previously reported in children by Wren et al. [14]. Indeed, the mean accuracy of these algorithms was reported to be $39 \%$ in previous studies [14], with the highest value being achieved by the Boersma algorithm (reported accuracy of 48\%). Our results are partly in line with these findings, but the mean accuracy of the 3 algorithms in our population was $62.23 \%$. The greatest accuracy $(71.7 \%)$, without any correction, was found using the Boersma algorithm, which was originally developed for use in children.

A practical implication of the present analysis is the division into 3 groups of locations around the mitral and tricuspid annuli: a left-sided group, a group of locations at risk of damaging the conduction system (AV block risk), and a right free-wall group (Figure 1). On considering these 3 subgroups, the algorithms by Arruda and Boersma seem to distinguish APs better than the one by Chiang (83.3\% and $84.1 \%$ vs. $67.5 \%)$. These results could be regarded as discordant with previous studies [13, 14], which reported that the algorithms developed in adults displayed low accuracy in children. Nevertheless, we only included patients with a structurally normal heart, who have some similar characteristics to adults with manifest ventricular pre-excitation.

The most important implication of our analysis is that integrating the 3 algorithms could dramatically improve their predictive power. Indeed, while inter-algorithm agreement in the study population ranged from good to fair when all 3 algorithms were concordant in predicting the need (or not) for a left-sided (trans-septal or retrograde aortic) access, their predictive power rose to $97.4 \%$ and $95.6 \%$. Similarly, the risk of AV block, which is considerable when the location of the pathway is right anterior, anteroseptal, or mid-septal, can be predicted or ruled out with $92.2 \%$ probability when the results of the 3 algorithms are integrated. This important finding could 


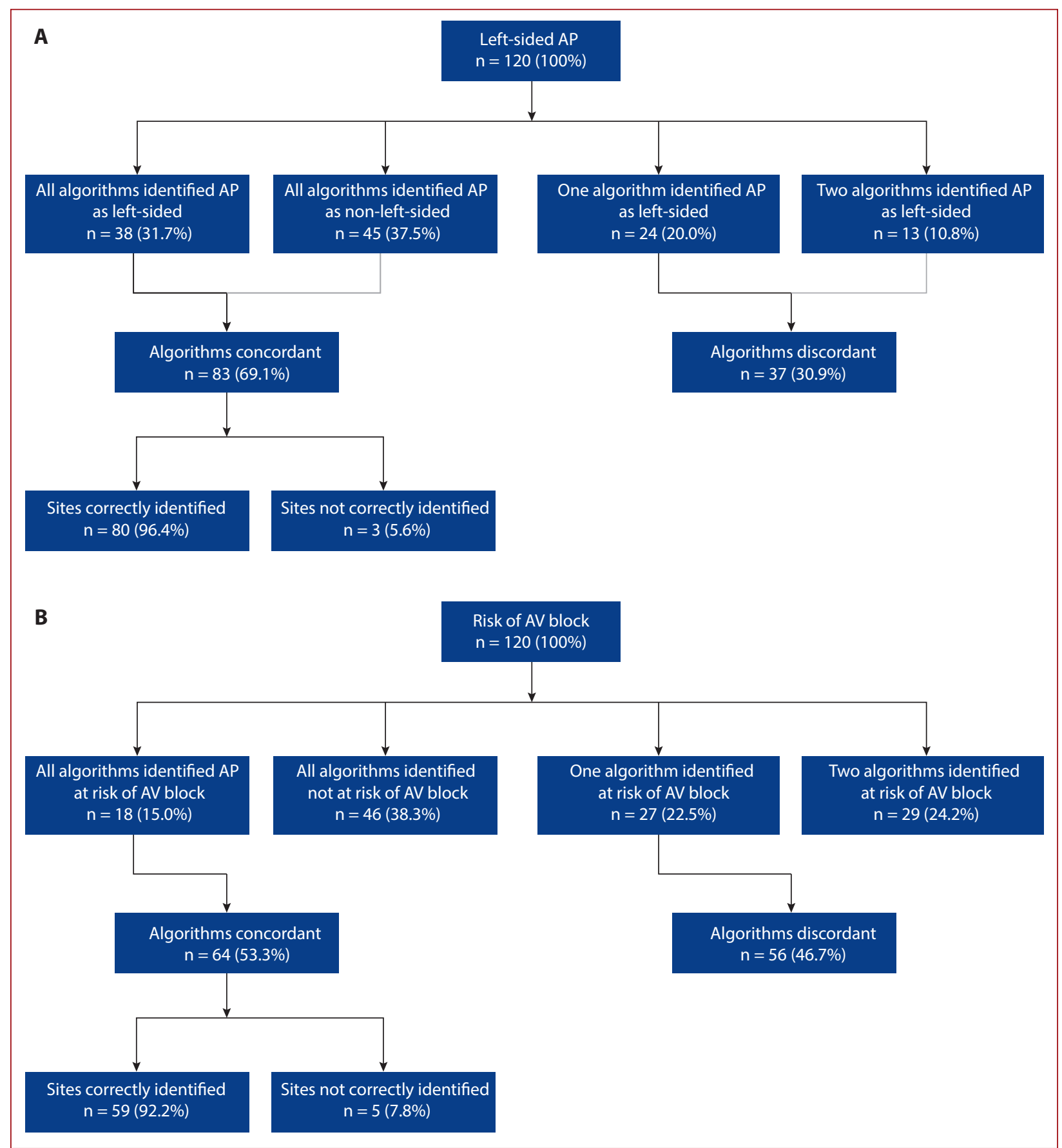

Figure 3. A step-down approach based on the concordance of the algorithms in identifying both left-sided sites (A) and sites at risk of an atrioventricular block (B)

Abbreviations: see Figure 1 and Table 3

help to plan accurately the procedure of ablation of the accessory pathway in terms of selection of vascular access, catheter choice, and the type of energy delivery. Moreover, specific tools, such as echocardiographic monitoring and anesthesiologist support, can be made promptly available during the procedure. Moreover, it is noteworthy that the improvement in prediction accuracy was not achieved by adding a difficult or novel tool, but by using well-known algorithms that are already used in everyday clinical practice. The proposed approach can be applied to a vast majority of cases of pre-excitation where concordance between algorithms is present, in our analysis $69 \%$ of cases.

In a recently published study, the authors claimed that the inaccuracy of published algorithms for predicting accessory pathways in children was due to differences in ECG parameters [14]. They proposed a new algorithm, which was validated in a prospective cohort, to overcome the limitations of the earlier algorithms in identifying septal locations. However, while grouping together every septal pathway (from anteroseptal to postero-septal) may reflect 
ECG vectors, it has a limited value when considering the practical need for a left-sided approach and the risk of AV block in small-sized pediatric patients.

The simple and powerful added value of combining more than one algorithm to predict pathway location could also help to guide therapeutic indications and discussion with parents during electrophysiological consultation. Since the possible implications (malignant arrhythmias) and adverse events related to an accessory pathway ablation could be devastating, a good precision in planning and estimating the procedural risk is more than important. Moreover, this has become more relevant in the recent era, when a lower threshold for prophylactic ablation is considered by some electrophysiologists [19].

Indeed, when discussing the indications for EPS and ablation with the parents/caregivers of a young child, it is important to provide them with a clear description of the procedure, an estimate of its duration, and its possible complications. In addition, the availability of a more detailed plan of ablation could be very useful, even to expert operators, when dealing with children of small size and low weight.

This study has some limitations. Its retrospective nature makes any conclusion driven by this analysis hypothesis-generating. Therefore, prospective data are needed in the future to strengthen the feasibility and accuracy of the proposed integrated approach. Patients with multiple accessory pathways were excluded given the reported inaccuracy of multiple non-invasive methods in locating more than one accessory pathway. Secondly, the gold standard used for AP location was the site of ablation, which, in children, offers less accurate spatial discrimination than other methods, such as autopsy or direct visualization during open-heart surgery. Patients were treated with radiofrequency or cryo-ablation according to operator preference, and this may result in a different risk of damage to cardiac structures; however, the study is limited to algorithms accuracy, and no complications were reported. Moreover, different numbers of possible locations and differences in the nomenclature of regions around the AV-valvular-annuli make the comparison between algorithms liable to some assumptions and inherent errors. However, Table 1 details how we overcame these peculiarities. Moreover, for this reason, grouping accessory pathways into 3 different regions seems to be an attractive approach. Another limitation can be the choice of the 3 algorithms we used in the study, these are surely not all published in this field, but we believe that a more inclusive approach could be misleading, and, therefore, we selected the 3 as a reference and proved higher accuracy than observed in previous studies. The last limitation of the proposed method is the fact that it is applicable only if the concordance of the 3 referred algorithms is achieved. In our study, the algorithms concordance was found in $69 \%$ of cases - a vast majority of the study population.

\section{CONCLUSIONS}

In our multicenter experience involving a pediatric population, an aggregated prediction score based on 3 reference algorithms yielded a high level of precision in predicting an accessory pathway location. This approach could be used to safely plan invasive procedures and to target ablation sites and may be a valuable alternative to a single-algorithm prediction strategy.

\section{Article information}

Conflict of interest: MM is an employee of Boston Scientific, Inc. Other authors declare no conflict of interest.

Open access: This article is available in open access under Creative Common Attribution-Non-Commercial-No Derivatives 4.0 International (CC BY-NC-ND 4.0) license, allowing to download articles and share them with others as long as they credit the authors and the publisher, but without permission to change them in any way or use them commercially. For commercial use, please contact the journal office at kardiologiapolska@ptkardio.pl.

\section{REFERENCES}

1. Brugada J, Katritsis DG, Arbelo E, et al. 2019 ESC Guidelines for the management of patients with supraventricular tachycardia. The Task Force for the management of patients with supraventricular tachycardia of the European Society of Cardiology (ESC). Eur Heart J. 2020; 41(5): 655-720, doi: 10.1093/eurheartj/ehz467, indexed in Pubmed: 31504425.

2. Brugada J, Blom N, Sarquella-Brugada G, et al. European Heart Rhythm Association, Association for European Paediatric and Congenital Cardiology. Pharmacological and non-pharmacological therapy for arrhythmias in the pediatric population: EHRA and AEPC-Arrhythmia Working Group joint consensus statement. Europace. 2013; 15(9): 1337-1382, doi: 10.1093/europace/eut082, indexed in Pubmed: 23851511.

3. Saul JP, Kanter R, Abrams D, et al. PACES/HRS expert consensus statement on the use of catheter ablation in children and patients with congenital heart disease. Heart Rhythm. 2016; 13(6): e251-e289, doi: 10.1016/j. hrthm.2016.02.009.

4. Arruda MS, McClelland JH, Wang X, et al. Development and validation of an ECG algorithm for identifying accessory pathway ablation site in Wolff-Parkinson-White syndrome. J Cardiovasc Electrophysiol. 1998; 9(1): 2-12, doi: 10.1111/j.1540-8167.1998.tb00861.x, indexed in Pubmed: 9475572.

5. Boersma L, García-Moran E, Mont L, et al. Accessory pathway localization by QRS polarity in children with Wolff-Parkinson-White syndrome. J Cardiovasc Electrophysiol. 2002; 13(12): 1222-1226, doi: 10.1046/j.15408167.2002.01222.x, indexed in Pubmed: 12521337.

6. Chiang $C E$, Chen SA, Teo WS, et al. An accurate stepwise electrocardiographic algorithm for localization of accessory pathways in patients with Wolff-Parkinson-White syndrome from a comprehensive analysis of delta waves and R/S ratio during sinus rhythm. Am J Cardiol. 1995; 76(1): 40-46, doi: 10.1016/s0002-9149(99)80798-x, indexed in Pubmed: 7793401.

7. d'Avila A, Brugada J, Skeberis V, et al. A fast and reliable algorithm to localize accessory pathways based on the polarity of the QRS complex on the surface ECG during sinus rhythm. Pacing Clin Electrophysiol. 1995; 18(9Pt 1): 1615-1627, doi: 10.1111/j.1540-8159.1995.tb06983.x, indexed in Pubmed: 7491305.

8. Fitzpatrick AP, Gonzales RP, Lesh MD, et al. New algorithm for the localization of accessory atrioventricular connections using a baseline electrocardiogram. J Am Coll Cardiol. 1994; 23(1): 107-116, doi: 10.1016/07351097(94)90508-8, indexed in Pubmed: 8277067.

9. Iturralde $\mathrm{P}$, Araya-Gomez V, Colin L, et al. A new ECG algorithm for the localization of accessory pathways using only the polarity of the QRS complex. J Electrocardiol. 1996; 29(4): 289-299, doi: 10.1016/s00220736(96)80093-8, indexed in Pubmed: 8913903.

10. Xie B, Heald SC, Bashir Y, et al. Localization of accessory pathways from the 12-lead electrocardiogram using a new algorithm. Am J Cardiol. 1994; 74(2): 161-165, doi: 10.1016/0002-9149(94)90090-6, indexed in Pubmed: 8023781. 
11. Pambrun T, El Bouazzaoui R, Combes N, et al. Maximal Pre-Excitation Based Algorithm for Localization of Manifest Accessory Pathways in Adults. JACC Clin Electrophysiol. 2018; 4(8): 1052-1061, doi: 10.1016/j. jacep.2018.03.018, indexed in Pubmed: 30139487.

12. Moskal P, Bednarski A, Kiełbasa G, et al. Increased preexcitation on electrocardiography improves accuracy of algorithms for accessory pathway localization in Wolff-Parkinson-White syndrome. Kardiol Pol. 2020; 78(6): 567-573, doi: 10.33963/KP.15378, indexed in Pubmed: 32438794.

13. Maden O, Balci KG, Selcuk MT, et al. Comparison of the accuracy of three algorithms in predicting accessory pathways among adult Wolff-Parkinson-White syndrome patients. J Interv Card Electrophysiol. 2015; 44(3): 213-219, doi: 10.1007/s10840-015-0057-6, indexed in Pubmed: 26384677.

14. Wren C, Vogel M, Lord S, et al. Accuracy of algorithms to predict accessory pathway location in children with Wolff-Parkinson-White syndrome. Heart. 2012; 98(3): 202-206, doi: 10.1136/heartjnl-2011-300269, indexed in Pubmed: 21917661.

15. Baek SM, Song MiK, Uhm JS, et al. New algorithm for accessory pathway localization focused on screening septal pathways in pediatric patients with Wolff-Parkinson-White syndrome. Heart Rhythm. 2020; 17(12): 2172 2179, doi: 10.1016/j.hrthm.2020.07.016, indexed in Pubmed: 32681992.
16. Teixeira CM, Pereira TA, Lebreiro AM, et al. Accuracy of the electrocardiogram in localizing the accessory pathway in patients with Wolff-Parkinson-White pattern. Arq Bras Cardiol. 2016; 107(4): 331-338, doi: 10.5935/abc.20160132, indexed in Pubmed: 27627222.

17. Bar-Cohen Y, Khairy P, Morwood J, et al. Inaccuracy of Wolff-Parkinson-white accessory pathway localization algorithms in children and patients with congenital heart defects. J Cardiovasc Electrophysiol. 2006; 17(7): 712-716, doi: 10.1111/j.1540-8167.2006.00467.x, indexed in Pubmed: 16836664.

18. Cohen J. A coefficient of agreement for nominal scales. Educational and Psychological Measurement. 1960; 20(1): 37-46, doi: 10.1177/001316446002000104.

19. Fleiss JL, Levin B, Paik MC. Statistical methods for rates and proportions, 3rd ed. John Wiley \& Sons, Hoboken 2003.

20. Moskal $P$, Jastrzębski $M$, Pitak $M$, et al. Malignant ventricular arrhythmias and other complications of untreated accessory pathways: an analysis of prevalence and risk factors in over 600 ablation cases. Kardiol Pol. 2020; 78(3): 203-208, doi: 10.33963/KP.15161, indexed in Pubmed: 31994490. 